EPJ Web of Conferences 108, 02011 (2016)

DOI: 10.1051/epjconf/201610802011

(C) Owned by the authors, published by EDP Sciences, 2016

\title{
Synthesis of the Thickness Profile of the Waveguide Layer of the Thin Film Generalized Waveguide Luneburg Lens
}

\author{
E. A. Ayryan ${ }^{1, a}$, G. D. Dashitsyrenov ${ }^{2, b}$, K. P. Lovetskiy ${ }^{2, c}$, and A. L. Sevastianov $2, d$ \\ ${ }^{1}$ Laboratory of Information Technologies, Joint Institute for Nuclear Research, Joliot-Curie 6, Dubna, \\ Moscow region, 141980, Russia \\ ${ }^{2}$ Department of Applied Probability and Informatics, Peoples' Friendship University of Russia, Miklukho- \\ Maklaya str. 6, Moscow, 117198, Russia
}

\begin{abstract}
A local variation in the thickness of the waveguide layer of integrated optics waveguide causes a local decrease of phase velocity, and hence bending of rays and of the wave front. The relationship of the waveguide layer thickness profile $h(y, z)$ with the distribution of the effective refractive index of the waveguide $\beta(y, z)$ is described in terms of a particular model of waveguide solutions of the Maxwell equations. In the model of comparison waveguides the support of the thickness irregularity of the waveguide layer $\Delta h$ coincides with the support of inhomogeneity of the effective refractive index $\Delta \beta$. A more adequate but more cumbersome model of the adiabatic waveguide modes allows them to mismatch supp $\Delta h \supset \operatorname{supp} \Delta \beta$. In this paper, we solve the problem of the $\Delta h$ reconstruction on the base of given $\Delta \beta$ of the thin film generalized waveguide Luneburg lens in a model of adiabatic waveguide modes. The solution is found in the form of a linear combination of Gaussian exponential functions and in the form of a cubic spline for the cylindrically symmetric $\Delta h(r)$ and in the form of a cubic spline for $\Delta \beta(r)$.
\end{abstract}

\section{Introduction}

The method of adiabatic waveguide modes proposed in $[1,2]$ reduces the boundary problem for a system of partial differential equations to the problem for a system of ordinary differential equations. The adiabatic waveguide modes of a smoothly irregular waveguide satisfy the exact boundary conditions on the inclined planes, tangent to the interface between two media, so that the system of ordinary differential equations for vertical distributions of the electromagnetic fields of the modes takes the form of two coupled oscillators for two polarizations of the electromagnetic field of the TE and TM modes. As an example, consider a dielectric waveguide of the following structure (figure 1).

In the regular part of the waveguide, a waveguide guided TE (TM) mode of the form

$$
\left(\begin{array}{c}
E_{y}^{j} \\
H_{x}^{j} \\
H_{z}^{j}
\end{array}\right)(x) \exp \left\{i \omega t-i \beta_{j} k_{0} z\right\}, \quad\left(\begin{array}{c}
E_{x}^{j} \\
E_{z}^{j} \\
H_{y}^{j}
\end{array}\right)(x) \exp \left\{i \omega t-i \beta_{j} k_{0} z\right\}
$$

\footnotetext{
ae-mail: ayrjan@jinr.ru

be-mail: genin_d@mail.ru

ce-mail: lovetskiy@gmail.com

$\mathrm{d}_{\mathrm{e}-\mathrm{mail}}$ : alsevastyanov@gmail.com
}

This is an Open Access article distributed under the terms of the Creative Commons Attribution License 4.0, which permits unrestricted use, distribution, and reproduction in any medium, provided the original work is properly cited. 


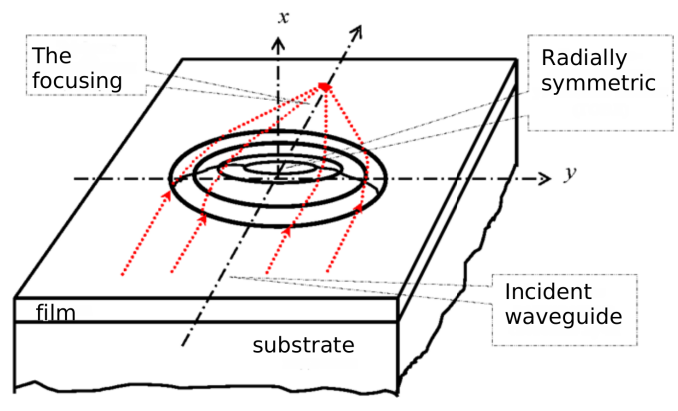

Figure 1. Schematic representation of (thin-film generalized waveguide lens) Luneburg TGWL

propagates along the axis $O z$ to infinity. In the vicinity of the origin, the waveguide mode runs across an irregularity of the waveguide formed by an additional waveguide layer with variable thickness $h(r)$ and cylindrically symmetric with respect to the axis $O x$. The phase velocity of the wave front (lines of constant phase) slows down (larger deceleration takes place closer to the origin, smaller at increasing distances from the origin), and two-dimensional "rays", locally orthogonal to the fronts, acquire local curvature toward the axis $O z$. If the profile $h(r)$ is selected properly, all the rays that have passed through the region of irregularity of the waveguide gather in the focal point on the axis $\mathrm{Oz}$ at a distance $F$ from the origin. Turning the incident beam of rays (the direction of the incoming regular waveguide mode) around the axis $O x$, we get an identical pattern: the focal point moves by the same angle along a "big" circle of radius $F$. Thus, the described irregularity of the waveguide forms the structure of a "Luneburg waveguide lens" that is, a two-dimensional Luneburg lens for the original waveguide mode of the regular waveguide.

\section{Luneburg lens}

The Luneburg lens was proposed by RK Luneburg in his course on the Maxwell optics [3]. It is described in terms of geometrical optics in a three-dimensional (or two-dimensional) space filled by a medium (or vacuum) with given permittivity and permeability, such that the optical density is uniform. In the vicinity of the origin inside the sphere (disk) of radius $R$ the optical density $n(r)$ (and therefore $\varepsilon(r), \mu(r))$ is not uniform, increasing toward the origin so that the parallel light beam after passing through the region of «n» inhomogeneity focuses on the distance $F \geq R$ from the origin of coordinates. If $F=R$ the Luneburg lens is called classical, it is realized provided

$$
n(r)=n_{c} \sqrt{2-(r / R)^{2}} .
$$

If $F>R$ the Luneburg lens is called generalized and $n(r)$ satisfies the integral relations [4]:

$$
n(r)=\exp [\omega(\rho, F)], \quad \text { where } \rho=r n(r) \quad \text { and } \quad \omega(\rho, F)=\frac{1}{\pi} \int_{\rho}^{\pi} \frac{\arcsin (x / F)}{\left(x^{2}-\rho^{2}\right)^{(1 / 2)}} d x .
$$

The angular aperture of the a classical Luneburg lens is $\Omega=180^{\circ}=\pi$. In the generalized Luneburg lens the angular aperture equals $\Omega=2 \operatorname{arctg}(R / F)$ and depends on the focal distance [7]. The shorter the focus, the larger the aperture, and $\lim _{F \rightarrow R} \Omega[F]=\pi$. 


\section{The waveguide Luneburg lens in a model of adiabatic waveguide modes in normalized variables}

The propagation of the electromagnetic radiation through a thin-film generalized waveguide Luneburg lens (TGWL) is described using adiabatic waveguide modes (AWM). The AWM model reduces the system of Maxwell equations with the boundary conditions at the interface between the two layers to two second order ordinary differential equations for the two components of the electromagnetic field of the AWM (with reduced boundary conditions) plus four algebraic expressions for the other four components of the electromagnetic field through two others and their derivatives. For a monochromatic radiation, the intensities of the electromagnetic field of waveguide radiation $(\mathbf{E}, \mathbf{H})^{T} \sim \exp \{i \omega t\}$ are described by special case mappings

$$
\begin{gathered}
(\mathbf{E}, \mathbf{H})^{T}: \mathbb{R}^{3} \times \mathbb{R} \rightarrow \mathbb{R}^{6}, \\
(x, y, z, t)^{T} \mapsto\left(E_{x}, E_{y}, E_{z}, H_{x}, H_{y}, H_{z}\right)^{T}(x, y, z, t)^{T} .
\end{gathered}
$$

Assuming an electromagnetic field of a fixed frequency $\omega$, we introduce the dimensionless "normalized" coordinates by setting the normalization $(x, y, z, t)^{T} \mapsto(\tilde{x}, \tilde{y}, \tilde{z}, \tilde{t})^{T}$, where $\tilde{x}=k_{0} x, \tilde{y}=k_{0} y$, $\tilde{z}=k_{0} z, \tilde{t}=\omega t$, with $k_{0}=\omega / c, c$ is the speed of light in vacuum, so that $\tilde{k}_{0}=1, \tilde{\omega}=1, \tilde{c}=1$ in new "normalized" coordinates (variables). The field intensities will then be measured in fractions of "amplitude" intensity of the incoming light flux

$$
\left(E_{j}, H_{k}\right) \mapsto\left(E_{j} / \sqrt{I}, H_{k} / \sqrt{I}\right) \equiv\left(\tilde{E}_{j}, \tilde{H}_{k}\right) .
$$

In terms of the normalized variables, the Maxwell equations take the form:

$$
\operatorname{rot} \tilde{\mathbf{H}}=i \tilde{\varepsilon} \tilde{\mathbf{E}}, \quad \operatorname{rot} \tilde{\mathbf{E}}=-i \tilde{\mu} \tilde{\mathbf{H}},
$$

where

$$
\begin{gathered}
\tilde{\mathbf{H}} \equiv \tilde{\mathbf{H}}_{\omega}(\tilde{x}, \tilde{y}, \tilde{z}, \tilde{t}), \quad \tilde{\mathbf{E}} \equiv \tilde{\mathbf{E}}_{\omega}(\tilde{x}, \tilde{y}, \tilde{z}, \tilde{t}), \\
\tilde{\varepsilon} \equiv \tilde{\varepsilon}_{\omega}(\tilde{x}, \tilde{y}, \tilde{z}) \equiv \varepsilon_{\omega}(x, y, z), \quad \tilde{\mu} \equiv \tilde{\mu}_{\omega}(\tilde{x}, \tilde{y}, \tilde{z}) \equiv \mu_{\omega}(x, y, z) .
\end{gathered}
$$

Tangential boundary conditions at the interface between two media can be written as:

$$
\left.\tilde{\mathbf{H}}^{\tau}\right|_{1}=\left.\tilde{\mathbf{H}}^{\tau}\right|_{2},\left.\quad \tilde{\mathbf{E}}^{\tau}\right|_{1}=\left.\tilde{\mathbf{E}}^{\tau}\right|_{2}
$$

At infinitely remote distance the asymptotic conditions for the tangential components of the electromagnetic field are:

$$
\left.\left\|\tilde{\mathbf{E}}^{\tau}\right\|\right|_{x \rightarrow \pm \infty}<+\infty,\left.\quad\left\|\tilde{\mathbf{H}}^{\tau}\right\|\right|_{x \rightarrow \pm \infty}<+\infty .
$$

The solutions of (1) for adiabatic waveguide modes are sought in the form:

$$
\left\{\begin{array}{c}
\tilde{\mathbf{E}}(\tilde{x}, \tilde{y}, \tilde{z}, \tilde{t}) \\
\tilde{\mathbf{H}}(\tilde{x}, \tilde{y}, \tilde{z}, \tilde{t})
\end{array}\right\}=\left\{\begin{array}{l}
\tilde{\mathbf{E}}(\tilde{x} ; \tilde{y}, \tilde{z}) \\
\tilde{\mathbf{H}}(\tilde{x} ; \tilde{y}, \tilde{z})
\end{array}\right\} \frac{\exp \{i \tilde{t}-i \tilde{\varphi}(\tilde{y}, \tilde{z})\}}{\sqrt{\tilde{\beta}(\tilde{y}, \tilde{z})}}
$$

where

$$
\tilde{\beta}_{y}(\tilde{y}, \tilde{z})=\left(\frac{\partial \tilde{\varphi}}{\partial \tilde{y}}\right), \quad \tilde{\beta}_{z}(\tilde{y}, \tilde{z})=\left(\frac{\partial \tilde{\varphi}}{\partial \tilde{z}}\right), \quad \text { and } \quad \tilde{\beta}(\tilde{y}, \tilde{z})=\sqrt{\tilde{\beta}_{y}(\tilde{y}, \tilde{z})^{2}+\tilde{\beta}_{z}(\tilde{y}, \tilde{z})^{2}} .
$$

Furthermore, the problem is reduced to a homogeneous system of linear algebraic equations for the vector of amplitude coefficients using the ODE solution expansion (in the zero approximation of the asymptotic method) over the fundamental system of solutions with undefined amplitude coefficients. 


\section{Waveguide fields and boundary conditions in the zero approximation}

In the multilayer waveguide, in the zero approximation, the equations for the longitudinal components of the electromagnetic field of the adiabatic waveguide modes take the form [5-7]:

$$
\begin{aligned}
& \frac{d^{2} \tilde{E}_{z}^{0}}{d \tilde{x}^{2}}+\left(\tilde{\varepsilon} \tilde{\mu}-\tilde{\beta}^{2}\right) \tilde{E}_{z}^{0}=0, \\
& \frac{d^{2} \tilde{H}_{z}^{0}}{d \tilde{x}^{2}}+\left(\tilde{\varepsilon} \tilde{\mu}-\tilde{\beta}^{2}\right) \tilde{H}_{z}^{0}=0 .
\end{aligned}
$$

The expressions for the other four components of the electromagnetic field take the form:

$$
\begin{aligned}
\tilde{H}_{x}^{0}=\frac{1}{\left(\tilde{\varepsilon} \tilde{\mu}-\tilde{\beta}_{z}^{2}\right)}\left(-i \tilde{\beta}_{z} \frac{d \tilde{H}_{z}^{0}}{d \tilde{x}}+\tilde{\varepsilon} \tilde{\beta}_{y} \tilde{E}_{z}^{0}\right), & \tilde{H}_{y}^{0}=\frac{1}{\left(\tilde{\varepsilon} \tilde{\mu}-\tilde{\beta}_{z}^{2}\right)}\left(-i \tilde{\varepsilon} \frac{d \tilde{E}_{z}^{0}}{d \tilde{x}}-\tilde{\beta}_{z} \tilde{\beta}_{y} \tilde{H}_{z}^{0}\right), \\
\tilde{E}_{x}^{0}=\frac{1}{\left(\tilde{\varepsilon} \tilde{\mu}-\tilde{\beta}_{z}^{2}\right)}\left(-i \tilde{\beta}_{z} \frac{d \tilde{E}_{z}^{0}}{d \tilde{x}}-\tilde{\mu} \tilde{\beta}_{y} \tilde{H}_{z}^{0}\right), & \tilde{E}_{y}^{0}=\frac{1}{\left(\tilde{\varepsilon} \tilde{\mu}-\tilde{\beta}_{z}^{2}\right)}\left(i \tilde{\mu} \frac{d \tilde{H}_{z}^{0}}{d \tilde{x}}-\tilde{\beta}_{z} \tilde{\beta}_{y} \tilde{E}_{z}^{0}\right) .
\end{aligned}
$$

In the subdomains (waveguide layers) with constant refractive indices $n_{j}^{2}=\varepsilon_{j} \mu_{j}$ the solutions $\tilde{E}_{z}^{0}$ of the fundamental system (3) are written as:

$$
\begin{aligned}
& \tilde{E}_{z}^{s}=\tilde{A}_{s} \exp \left\{\tilde{\gamma}_{s}\left(\tilde{x}-\tilde{a}_{1}\right)\right\}, \quad \tilde{E}_{z}^{f}=\tilde{A}_{f}^{+} \exp \left\{i \tilde{\chi}_{f}\left(\tilde{x}-\tilde{a}_{2}\right)\right\}+\tilde{A}_{f}^{-} \exp \left\{-i \tilde{\chi}_{f}\left(\tilde{x}-\tilde{a}_{2}\right)\right\}, \\
& \tilde{E}_{z}^{l}=\tilde{A}_{l}^{+} \exp \left\{i \tilde{\chi}_{l}\left(\tilde{x}-\tilde{a}_{2}-\tilde{h}\right)\right\}+\tilde{A}_{l}^{-} \exp \left\{-i \tilde{\chi}_{l}\left(\tilde{x}-\tilde{a}_{2}-\tilde{h}\right)\right\}, \quad \tilde{E}_{z}^{c}=\tilde{A}_{c} \exp \left\{-\tilde{\gamma}_{c}\left(\tilde{x}-\tilde{a}_{2}-\tilde{h}\right)\right\} .
\end{aligned}
$$

while the solutions $\tilde{H}_{z}^{0}$ of (4) in the form:

$$
\begin{aligned}
& \tilde{H}_{z}^{s}=\tilde{B}_{s} \exp \left\{\tilde{\gamma}_{s}\left(\tilde{x}-\tilde{a}_{1}\right)\right\}, \quad \tilde{H}_{z}^{f}=\tilde{B}_{f}^{+} \exp \left\{i \tilde{\chi}_{f}\left(\tilde{x}-\tilde{a}_{2}\right)\right\}+\tilde{B}_{f}^{-} \exp \left\{-i \tilde{\chi}_{f}\left(\tilde{x}-\tilde{a}_{2}\right)\right\}, \\
& \tilde{H}_{z}^{l}=\tilde{B}_{l}^{+} \exp \left\{i \tilde{\chi}_{l}\left(\tilde{x}-\tilde{a}_{2}-\tilde{h}\right)\right\}+\tilde{B}_{l}^{-} \exp \left\{-i \tilde{\chi}_{l}\left(\tilde{x}-\tilde{a}_{2}-\tilde{h}\right)\right\}, \quad \tilde{H}_{z}^{c}=\tilde{B}_{c} \exp \left\{-\tilde{\gamma}_{c}\left(\tilde{x}-\tilde{a}_{2}-\tilde{h}\right)\right\} .
\end{aligned}
$$

Substituting (6)-(7) into (5), we obtain expressions for the other four components of the electromagnetic field. The substitution of all of them in the boundary equations (2) will result in expressions which, after reducing similar terms, give a system of homogeneous linear algebraic equations for the undefined amplitude coefficients $\tilde{A}_{s}, \tilde{B}_{s}, \tilde{A}_{f}^{ \pm}, \tilde{B}_{f}^{ \pm}, \tilde{A}_{l}^{ \pm}, \tilde{B}_{l}^{ \pm}, \tilde{A}_{c}, \tilde{B}_{c}$. This system which is characterized by a matrix $M(\beta)$, can have non-trivial solutions provided [6,8]

$$
\operatorname{det} M(\beta(r))=0 \text {. }
$$

\section{A thin-film generalized waveguide Luneburg lens synthesis}

The solution of a given nonlinear PDE of the first order with respect to $h(r)$ resolves the proper profile of the Luneburg TGWL.

Southwell [9] has solved the thickness profile of a Luneburg TGWL with parameters $n_{s}=1.47$, $n_{f}=1.565, n_{l}=2.10, n_{c}=1.0, d=1.0665 \mu, \lambda=0.5 \mu$ by the method of comparison waveguides in which the precise boundary conditions for the Maxwell equations are replaced by their approximations - projections on the horizontal plane [10]. We repeated these calculations for the focal lengths given in [9]. The support of the irregularity region of TGWL in the method of comparison waveguides automatically coincides with the support of $n_{\mathrm{eff}}^{j}(r)=\beta_{j}(r) / \beta_{j}$ inhomogeneity of the two-dimensional TGWL of Luneburg: $\operatorname{supp} h=\operatorname{supp} n=\operatorname{supp} \beta$.

The method of adiabatic waveguide modes does not require the matching of these two supports. However, the fact that the irregularity of TGWL forces to focus a family of curves locally orthogonal 
to the focused wave front of the waveguide mode, implies that the region of the waveguide irregularity does not exceed the circle $Q(F)$ of radius F: supp $h \subseteq Q(F)$. Following this reasoning, we seek the thickness profile of the irregular waveguide layer $\mathrm{h}(\mathrm{r})$ in a circle of radius $\mathrm{F}$. The equation (8) of the solution of the thickness profile of the irregular waveguide layer of Luneburg TGWL depends on the trajectories of the rays (parallel at the entrance to the irregularity region) and on their velocities derived from the equations for the rays that pass through the region of inhomogeneity. From the ODE systems for $(y, z)^{T}(s)$

$$
\frac{d}{d s}\left(\beta(y, z) \frac{d y}{d s}\right)=\frac{\partial \beta}{\partial y}(y, z), \quad \frac{d}{d s}\left(\beta(y, z) \frac{d z}{d s}\right)=\frac{\partial \beta}{\partial z}(y, z),
$$

using the substitutions $\frac{d y}{d z}=V, A=\frac{1}{\beta} \frac{\partial \beta}{\partial z}, B=\frac{1}{\beta} \frac{\partial \beta}{\partial y}$, we obtain the equivalent system of ODEs

$$
\frac{d y}{d z}=V, \quad \frac{d V}{d z}=\left(1+V^{2}\right)(B-A V) .
$$

The Cauchy problem for the system (8) with the initial conditions

$$
y\left(z_{0}\right)=y_{0}, \quad V\left(z_{0}\right)=0,
$$

is solved by the Runge-Kutta-Fehlberg method of the 6th order with automatic step selection resulting in the family of "data" $y_{j}^{F}\left(z_{k}^{j}\right), V_{j}^{F}\left(z_{k}^{j}\right)$ at the family of points automatically generated during the derivation of the solution of the problem (9)-(10) for any beforehand defined focal distance F. Data from the files $y_{j}^{F}\left(z_{k}^{j}\right), V_{j}^{F}\left(z_{k}^{j}\right)$ allows the calculation of the components of the vector field of the phase delay $\vec{\beta}=\left(\beta_{z}, \beta_{y}\right)^{T}$ of the AWM for which $\beta(y, z)=\sqrt{\beta_{y}^{2}+\beta_{z}^{2}}$ from

$$
\beta_{y}\left(z_{k}^{j}\right)=\beta\left(z_{k}^{j}\right) V\left(z_{k}^{j}\right) /\left(1+V^{2}\left(z_{k}^{j}\right)\right)^{1 / 2}, \quad \beta_{z}\left(z_{k}^{j}\right)=\beta\left(z_{k}^{j}\right) /\left(1+V^{2}\left(z_{k}^{j}\right)\right)^{1 / 2} .
$$

The result (11) allows to complement the previously generated data file to $y_{j}^{F}\left(z_{k}^{j}\right), V_{j}^{F}\left(z_{k}^{j}\right), \beta_{y}^{F}\left(z_{k}^{j}\right)$, $\beta_{z}^{F}\left(z_{k}^{j}\right)$. Now we have at our disposal all the necessary data for the formulation of the problem of synthesis $h^{F}(r)$. The matrix depends on the following variables

$$
\begin{gathered}
\widehat{M}\left(\beta^{F}\right)=\widehat{M}\left(n_{s}, n_{f}, n_{l}, n_{c}, d,\left\{z_{k}^{j}, y_{k}^{j}, V_{k}^{j}\right\}, \beta^{F}\left(y_{k}^{j}, z_{k}^{j}\right), \gamma_{s}^{F}\left(y_{k}^{j}, z_{k}^{j}\right), \gamma_{c}^{F}\left(y_{k}^{j}, z_{k}^{j}\right), \chi_{f}^{F}\left(y_{k}^{j}, z_{k}^{j}\right), \chi_{l}^{F}\left(y_{k}^{j}, z_{k}^{j}\right),\right. \\
\left.\beta_{y}^{F}\left(y_{k}^{j}, z_{k}^{j}\right), \beta_{z}^{F}\left(y_{k}^{j}, z_{k}^{j}\right), h^{F}\left(z_{k}^{j}, y_{k}^{j}\right), \frac{\partial h^{F}}{\partial y}\left(z_{k}^{j}, y_{k}^{j}\right), \frac{\partial h^{F}}{\partial z}\left(z_{k}^{j}, y_{k}^{j}\right), F\right) .
\end{gathered}
$$

For the matrix $\widehat{M}\left(*, z_{k}^{j}, y_{k}^{j}, \ldots, F\right)$ at each point of the "phase-ray mesh $\left\{z_{k}^{j}, y_{k}^{j}\right\}^{F}$ " the condition

$$
\operatorname{det} \widehat{M}\left(*, z_{k}^{j}, y_{k}^{j}, \ldots, F\right)=0
$$

has to be fulfilled. The approximation $h_{N}{ }^{F}(r)$ of the function $h^{F}(r)$ which defines the thickness profile of the irregular waveguide layer, is sought in the form:

$$
h_{N}^{F}(z, y)=\sum_{i=1}^{N} K_{i} \exp \left\{-\frac{\left(y-y_{c}\right)^{2}+\left(z-z_{c}\right)^{2}}{C_{i}^{2}}\right\}
$$


which allows the explicit calculation of the derivatives. The search for the unknown coefficients $\left(K_{i}, C_{i}\right)$ to construct an approximate solution $h^{F}(r)$ in (12) is carried out by minimizing the objective function

$$
F_{\text {arg }}\left(K_{i}, C_{i}\right)=\sum_{z_{k}^{j}, y_{k}^{j}}\left|\operatorname{det} \widehat{M}\left(h_{N}^{F}(z, y), \frac{\partial h_{N}^{F}(z, y)}{\partial y}, \frac{\partial h_{N}^{F}(z, y)}{\partial z}\right)\right|^{2}
$$

by the Nelder-Mead method [8].

\section{Conclusion}

The paper describes the classical and generalized Luneburg lens in bulk and waveguide implementation. The link between the focusing inhomogeneity of the effective refractive index of the waveguide Luneburg lens and the irregularity of the waveguide layer thickness generating this inhomogeneity is demonstrated. For the dispersion relation of the irregular thin-film waveguide in the AWM model the problem of the "mathematical" synthesis and computer-aided design of the waveguide layer thickness profile for Luneburg TGWL with a given focal length is solved.

The calculations are carried out in normalized (in a special way) coordinates in order to adapt the used relations to computer calculations.

The obtained solution is compared with the same solution within the model of comparison waveguides, which is rougher as compared to the AWM model due to the replacement of the exact boundary conditions (for the tangential components of the electromagnetic field) by their projections onto the horizontal plane.

\section{Acknowledgements}

The work is partially supported by RFBR grants No's 13-01-00595 and 14-01-00628.

\section{References}

[1] L.A. Sevastianov and A.A. Egorov, Opt. Spectrosc. 105, 576 (2008)

[2] A.A. Egorov and L.A. Sevast'yanov, Quantum Electronics 39 (6) 566-574 (2009)

[3] R.K. Luneberg, Mathematical theory of Optics (University of California Press, 1966)

[4] S.P. Morgan, J. Appl. Phys. 29, 9, 1358-1368 (1958)

[5] E.A. Ayryan, A.A. Egorov, L.A. Sevastianov, K.P. Lovetskiy, and A.L. Sevastyanov, Lecture Notes in Computer Science 7125, 136-147 (2012)

[6] A.L. Sevastyanov, Lett. PEPAN 8, 804-811 (2011)

[7] A.A. Egorov, L.A. Sevastianov and A.L. Sevastyanov, Quantum Electronics 44 (2), 167 (2014)

[8] A.A. Egorov, A.L. Sevastyanov, E.A. Ayryan, and L.A. Sevastianov, Matem. Model. 26, 11, 37-44 (2014)

[9] Southwell W.H.JOSA 67, 8, 1010-1014 (1977)

[10] L.A. Sevastianov, A.A. Egorov, and A.L. Sevastyanov, Physics of Atomic Nuclei 76, 2, 224 $239(2013)$ 